\title{
MUJERES Y
}
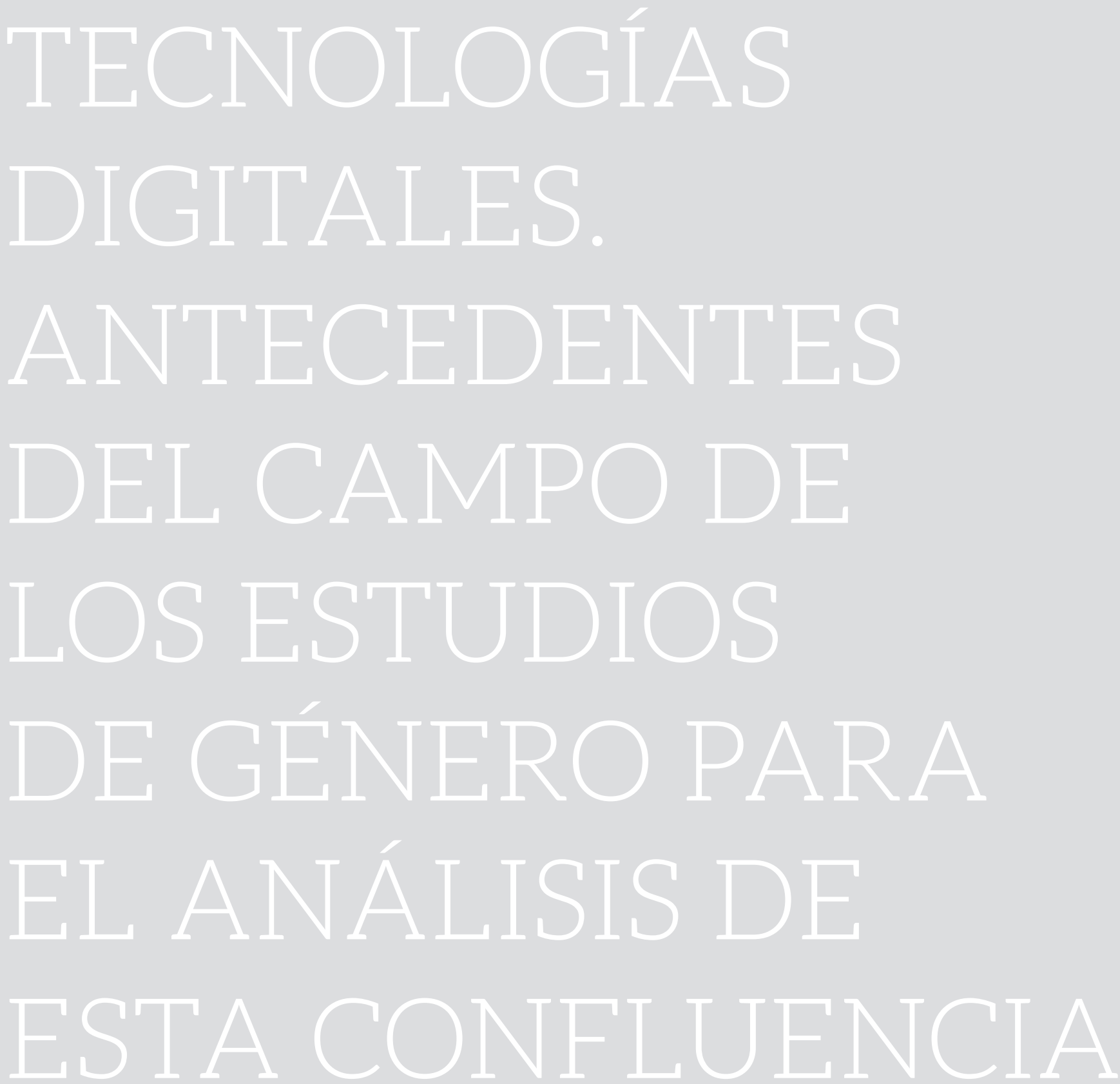

[ ARTIGO ]

Veronica Sofia Ficoseco

$d b_{\text {EXTPAPRENSA }}$ 


\section{[ RESUMO ABSTRACT RESUMEN]}

Este artigo apresenta e sistematiza linhas de pesquisa com perspectiva de gênero que abordam a questão das relações sociais e a tecnologia a partir de enfoques que tornam visíveis e problematizam o lugar e a situação das mulheres em cada área, bem como a manifestação que as relações de gênero adquirem - como relações de poder - quando são analisadas do ponto de vista da tecnologia. Nos concentramos em tecnologias digitais específicamente, considerando por um lado sua materialidade e, por outro lado, o espaço de relações mediado que criam. As linhas de pesquisa são apresentadas sistematicamente e agrupadas em três pontos de análise de acordo com a forma como a relação entre tecnologia e sociedade é concebida em cada uma delas.

Palavras-chaves: Estudos de gênero. Estudos sociais da tecnologia. Tecnologias digitais.

This article presents and systematize research lines with a gender perspective that has addressed the issue of social relations with technology from approaches that make visible and problematize the place and the situation of women in each area, as well as the modalities that gender relations acquire -as power relationships- when the approach is linked with technology. We focus specifically on digital technologies, considering, on the one hand, their materiality and, on the other, sorts of mediated relationships they enable. The research lines are presented systematically grouped in three analytic lines according to the way the link between technology and society is conceived in each.

Keywords: Social movements. Folkcommunication. Social media. Web 2.0. Mediaactivism.

En este artículo se exponen y sistematizan algunas líneas de investigación con perspectiva de género que abordaron la temática de las relaciones sociales con la tecnología desde enfoques que visibilizan y problematizan el lugar y la situación de las mujeres en cada ámbito, como así también las modalidades que adquieren las relaciones de género -en tanto relaciones de poder- cuando éstas son analizadas través de vínculos con la tecnología. Nos concentramos específicamente en tecnologías digitales, considerando, por un lado, su carácter empírico material y, por otro, el espacio de relacionamiento mediado que habilitan. Los antecedentes se presentan agrupados sistemáticamente en tres líneas analíticas definidas de acuerdo a la manera en que es concebida la relación entre tecnología y sociedad.

Palabras clave: Estudios de género. Estudios sociales de la tecnología. Tecnologías digitales. 
En este artículo se presenta de modo analítico y sistematizado, un breve recorrido a través de algunos de los antecedentes y aportes realizados desde diversas áreas de los estudios de género a la construcción de conocimiento académico sobre las relaciones de las mujeres y las tecnologías, enfocando específicamente a las tecnologías digitales o TICs.

A tal fin se construyó un recorrido sistemático que atraviesa trabajos académicos y líneas de investigación que, como conjunto, tienen en común el hecho de reflexionar en torno a las relaciones sociales con las tecnologías desde una perspectiva de género. La variedad de los mismos alcanza diversos tópicos, como por ejemplo: el lugar que las tecnologías ocupan en nuestras sociedades e imaginarios contemporáneos; la manera en que desde diversas políticas públicas se estimula la inclusión digital de la población y el lugar que ocupan las mujeres en esas políticas; las nuevas modalidades de trabajo, de militancia y de relaciones sociales y afectivas propiciada por las tecnologías digitales; la inscripción que hacen las mujeres de estas tecnologías en su vida cotidiana; los modos particulares que presentan las mujeres -al igual que cualquier población o grupo constituido socialmente como minoría- de transitar entornos mediados; la relación de estas nuevas prácticas con las relaciones de género y los históricos estereotipos que marcan las posibilidades y expectativas de las mujeres, entre otros temas.

La sistematización de antecedentes presentada este artículo forma parte del armazón teórico, epistemológico y metodológico de una tesis doctoral defendida en la Universidad Nacional de La Plata (Argentina). Entendemos que la utilidad de los recorridos y análisis exhaustivos de antecedentes radica en que habilitan la posibilidad de inscribir de modo solvente un trabajo de investigación en un campo académico de referencia.

En el caso del presente artículo, el texto se centra en la presentación tanto de antecedentes clásicos como de trabajos de investigadoras latinaomericanas en el incipiente campo académico de los estudios feministas de las tecnologías. El trabajo de sistematización tiene también el objetivo de visibilización de fructíferas y rigurosas líneas de investigación y antecedentes desarrolladas durante las últimas tres décadas, las cuales sin embargo no suelen gozar de amplia difusión y reproducción en los circuitos académicos de mayor concentración de prestigio y de eficacia simbólica para nombrar y establecer los términos en que las tecnologías digitales son estudiadas.

Las investigaciones consideradas se agrupan en tres líneas analíticas definidas de acuerdo a la manera en que es concebida la relación entre tecnología y sociedad en cada una de ellas: En la primera línea se agrupan investigaciones basadas en la consideración de las tecnologías digitales y los ámbitos mediados tecnológicamente como desarticuladores de las coordenadas clásicas que ordenan la vida social, como las de tiempo y espacio, y por lo tanto los definen como campos propicios para la liberación de los estereotipos sociales relacionados con el cuerpo sexuado y la inauguración de nuevas formas de participación, reconocimiento y construcción de identidades; en la segunda línea se sistematizan trabajos inscriptos en la perspectiva de las brechas, especialmente aquellos que a partir del concepto de la brecha digital de género, problematizan 
ROLES Y ESTEREOTIPOS

los modos en que se dirime el acceso, uso y apropiación de conocimientos y de herramientas en el campo tecnológico; por último, en una tercera línea, se agrupan investigaciones que toman como base la coproducción entre tecnología y sociedad, los cuales buscan dar cuenta de procesos históricos y estructurales que permiten ubicar al género como un elemento esencial en el sostenimiento y reproducción de los sistemas sociotécnicos contemporáneos.

El recorrido que se presenta es premeditadamente no cronológico, los trabajos citados se organizan en función de ejes en común encontrados en sus planteos analíticos, en sus bases epistemológicas, o por su confluencia sobre materiales empíricos similares desde perspectivas diversas.

Esta recopilación sistemática persigue dos objetivos principales: el primero de ellos es dar cuenta de la riqueza y variedad de los aportes que los estudios de género han realizado al campo de los estudios sociales de las tecnologías. Por otra parte, también se propone aportar a la conformación de una base de antecedentes que resulten operativos para repensar una serie de preguntas e interrogantes de características locales latinoamericanas en el amplio contexto de dichas líneas teóricas y de investigación. ENTORNOS MEDIADOS POR TECNOLOGÍAS

\begin{abstract}
A partir de la década de los '90, inspiradas principalmente por la obra de Donna Haraway (1991, 1998), tomaron forma nuevas líneas de problematización de las relaciones sociales con las tecnologías inscriptas en los estudios de género.
\end{abstract}

Entre estas líneas se encuentran trabajos de investigadoras que interpretaron que por sus características, internet -tomado como paradigma de los entornos mediados por tecnologías digitales- propiciaría una especie de liberación o laxitud de las normativas sociales que marcan las diferencias entre los cuerpos, y por lo tanto, las diferencias entre géneros. Esto se debía a la concepción generalizada de que frente a los cambios sociales y desplazamientos de las relaciones característicos de la era digital, muchas de las barreras o condicionamientos que imponía la corporalidad, comenzarían a influir menos o de modo menos determinante en las relaciones sociales, por lo que tópicos como la construcción de identidades de género alternativas o la toma de la palabra y la acción por parte de sectores antes relegados de la arena pública -por ejemplo las mujeres- se volvían más plausibles.

Cabe señalar que estos planteos se desenvolvieron aparentemente sin problematizar ni desnaturalizar de modo explícito los estereotipos de la diferencia en la dicotomía hombre/mujer, asignándole un valor incluso ventajoso a lo que antes representaba una posición de subordinación. Es decir que desde esta perspectiva se coloca en un lugar de mayor valor a las características entendidas tradicionalmente como femeninas. Es 
el caso de algunas formulaciones estereotipadas -cuya naturalización es muy discutida desde el feminismocomo por ejemplo la afirmación de la capacidad de las mujeres para realizar múltiples tareas al mismo tiempo, la aptitud femenina para la comunicación, la empatía con otras personas, entre otras.

Ejemplos de este enfoque son los trabajos de la británica Sadie Plant (1998). Esta autora contempla las tecnologías digitales como un elemento definitivo a tener en cuenta para la liberación de las mujeres, sentando la tendencia analítica de enfocar la apropiación de las tecnologías digitales y los usos del ciberespacio como terrenos de lucha por las reivindicaciones de género. Esta tendencia, a su vez, proporcionó en gran medida las bases de lo que se conoció como la base académica del ciberfeminismo.

Esa línea de pensamientoacción, el ciberfeminismo, cuyos inicios se remontan a la década de los '90, presenta algunos puntos de contacto con lo que se denominó la segunda ola del feminismo. Uno de estos puntos comunes es que toma como base la diferencia biológica entre hombre y mujer como dato cierto y a partir de allí se articulan los procesos de visibilización de las inequidades y violencias en las relaciones generizadas, especialmente de las relaciones de género en relación con las tecnologías en general. Al mismo tiempo, se construyen propuestas de disputa de los sentidos hegemónicos de los procesos de cambio tecnológico y el lugar de las mujeres en los mismos.

Una característica destacada de esta línea teórica es, como se mencionó anteriormente, que construye sus reflexiones en torno a estereotipos clásicos y opresivos de la feminidad normativa -la mujer como madre, su reclusión al ámbito privado, la experiencia del lenguaje como ajeno, la fragilidad, la sumisión de carácter, la tendencia a la emotividad, la habilidad natural para las tareas de cuidado y reproducción y la también natural inexperticia para lo relacionado con la razón y la técnica -. Nos interesa especialmente destacar entre sus bases a la presuposición de la relación lejana o negativa entre mujeres y tecnologías digitales. Estos estereotipos antes mencionados, a los que desde esta perspectiva teórica se considera resabios o remanentes de otros órdenes sociales ubicados temporalmente en un estadío del capitalismo no digitalizado, son resituados en el tecno capitalismo actual resignificándolos como características sociales que colocarían a las mujeres en una posición ventajosa frente a los cambios que las tecnologías traen aparejados (Plant, 1998).

Entonces, si las mujeres en la historia occidental reciente han sido ubicadas por las normativas sociales en el ámbito de lo doméstico, han ocupado los puestos de trabajo remunerado más precarios, inestables y peor remunerados, han sido socializadas para desarrollar preferentemente sus aptitudes para el establecimiento de relaciones y comunicación con los demás, esto derivaría en que ante los cambios en el mercado laboral, económico y de los afectos que se iniciaron hace cuatro décadas, con la creciente flexibilización laboral, los puestos de trabajo domiciliario o a distancia y la virtualización del conocimiento, los consumos culturales y las relaciones sociales, las mujeres no se vieran sorprendidas ya que se trata de su modo habitual de inserción social. Más aún, las mujeres se encontrarían comparativamente mejor preparadas que los hombres para insertarse en los entornos laborales y en los espacios de sociabilidad mediado por tecnologías. Este dato por sí 
mismo resultaría, desde la perspectiva ciberfeminista, alentador para plantear la oportunidad histórica del feminismo para cambiar las estructuras sociales occidentales (Id., 1998).

En una línea cercana, Allucquére Stone (1999), desde una inscripción ciberfeminista en los estudios queer, explora las complejidades de las construcciones del deseo, el género y la corporalidad online, es decir, en entornos mediados tecnológicamente También en sintonía con este enfoque, podemos mencionar las investigaciones referidas a las construcciones de identidades de género no normativas y sexualidades disidentes en entornos virtuales (De Abreu, 2010).

Estas heterogéneas líneas de pensamiento, investigación y - por qué no - militancia, fueron inscriptas transversalmente en las investigaciones que se abocaron a analizar los nuevos roles de las mujeres en escenarios sociales tecnologizados. Se destacan en esta línea los trabajos de Rossi Braidotti (2002) y Celia Amorós (2008), quienes mediante análisis de corte histórico y cultural de los cambios materiales y políticosdelasúltimasdécadas, sostienen que estaríamos frente a la oportunidad de las mujeres de constituirse en sujetos de cambio mediante la construcción de un rol femenino en la sociedad de la información que no quede al margen de las nuevas posibilidades y de inserciones más exitosas y menos inequitativas en los procesos centrales de las estructuras productivas y culturales actuales.

Una de las principales líneas en que se plantea la voluntad de visibilización de las inequidades y de resignificación de las mismas encarada por el ciberfeminismo, es la discusión en torno a la corporalidad y los modos de ser y de representar a los cuerpos de mujeres en relación con las tecnologías y los espacios mediados. Para ello se plantea la concepción de los cuerpos desde el punto de vista de la encarnación, es decir de cuerpos múltiples o de conjuntos de posiciones corpóreas. Esto trajo aparejadas algunas implicaciones epistemológicas notables, entre ellas las concepciones del cuerpo en el ciberespacio como situado en espacio y tiempo múltiples, capaces de llevar a cabo combinaciones e interacciones multisituadas y multilocalizadas. La subjetividad corporeizada, entendida de ese modo, se opone de modo directo a los imaginarios occidentales más clásicos que instauraron la concepción de la participación en situaciones y vínculos mediados como descorporización (Braidotti, 2002).

En igual sentido, son diversos los trabajos de investigadoras alineadas en el ciberfeminismo que han analizado las contradicciones e inequidades implícitas en los imaginarios tecnológicos contemporáneos, los cuales popularizan imágenes de lo virtual como campos incorpóreos y sin sexos, mientras que en el mismo movimiento se reproducen algunas de las imágenes más normativas de la identidad sexual, como así también de clase y étnicas.

La cuestión principal a la que se aproximan las diversas autoras en este campo, es el develamiento de posibles líneas de concepción política y de intervención institucional y legal respecto del desajuste entre aquello que los imaginarios tecnológicos dominantes postulan acerca de virtualidad o experiencia mediada y lo que efectivamente ocurre para aquellos agentes subalternizados, especialmente las mujeres. Investigaciones relativas a la violencia hacia las mujeres a través de las tecnologías digitales, las diferencias de oportunidades en los entornos online y los estudios del lenguaje mediado, son característicos de estas líneas (Id. 2002; Rubio Liniers, 2003; Martínez Collado, 2008; Natansohn y Schuch Brunet, 2009). 
Una de las líneas de mayor difusión en los estudios sociales de las tecnologías y su enfoque desde los estudios de género, es la que explora la relación de las mujeres y la tecnología desde la perspectivadelasbrechas. Enestecasose trata de lo que se nombra como la brecha cualitativa de género. Cabe señalar que el concepto mismo de segunda brecha digital de género, implica tomar como punto de partida y a la vez como objetivo la problematización de las razones de la predominante presencia masculina en las áreas estratégicas de la educación, el trabajo y la investigación relacionada con las TICs (Castaño, 2009).

Algunas perspectivas académicas de análisis social de las tecnologías, especialmente las agrupadas en torno al paradigma de las brechas, sostienen que en el actual mundo globalizado los indicadores más relevantes del estado de inclusión social de las personas se relacionan con el grado de capacidad de utilizar las TICs para la realización de tareas y actividades entendidas por esta línea de pensamiento como productivas o significativas por la complejidad procedimental que revisten y por el grado de inclusión en los circuitos de la sociedad digital que implican, estas son: la e-educación, el e-trabajo, el e-gobierno y el e-comercio (Castaño, 2008).

Las líneas principales de indagación de las investigaciones así planteadas indagan en las políticas públicas, datos de empresas privadas, datos censales, relevamientos, instituciones, entre otras fuentes, en busca de definir la evolución de la inclusión material -tanto en términos de adquisición o acceso a equipos como de conocimientos- de las mujeres en el uso y consumo de tecnologías digitales.
Lo que se busca principalmente es comprender la manera en que diferentes políticas, diversos contextos, implican diferentes espacios de participación y oportunidades para las mujeres en un nuevo modelo de sociedad que se pretende universal para todos y todas. Es el caso de los trabajos de Natansohn (2013); Castaño (2008); Castaño, Martín y Vázquez (2008); Vázquez y Añino (2008), Sainz y González (2008) y Sallé (2008).

En cuanto al análisis de las políticas que pretenden garantizar el acceso de las clases populares a ciertos consumos tecnológicos, destacamos abordajes centrados en caracterizar los esfuerzos y acciones que varían de acuerdo al contexto pero que en general se caracterizan por políticas de inversiones en infraestructura, investigación, ampliación del acceso a los servicios de telecomunicaciones, entre otras (Lago Martínez, 2005, 2011), en la misma línea, esa batería de políticas es desglosada y se llama la atención sobre la nula presencia de la mujer como destinataria (Finquelievich y Lago Martínez, 2003).

Este enfoque, si bien útil para desnaturalizar las desigualdades empíricas implícitas en cualquier afirmación universal sobre la inclusión, no suele reflexionar en torno a la contradicción epistemológica que implica la pretensión de ofrecer las mismas oportunidades construidas en clave universal a grupos que no han sido socializados de la misma manera, ni ocupando los mismos lugares, ni accediendo a los mismos territorios, bienes, acciones, derechos, entre otros. Es decir, la dimensión de la diferencia se encuentra ocluida desde enfoques de este tenor, afirmación que de ninguna manera implica desconocer el valor que estas perspectivas adquieren en los enfoques tendientes a la planificación y a la intervención socio política. 


\section{LA TRANSVERSALIDAD DEL GÉNERO EN LA RELACIÓN TECNOLOGÍA / SOCIEDAD}

El reconocimiento de la relación de coproducción entre tecnología y sociedad implicó la posibilidad teórica de integrar la dimensión de género, ya entendida como factor constitutivo e inherente a todas las relaciones sociales, al análisis de los sistemas sociotécnicos, no como factor marginal o dimensión complementaria, sino como elemento constitutivo y modelador de dichos sistemas.

En ese marco diversas autoras han destacado la importancia de observar las diferencias estructurales en la apropiación de las herramientas tecnológicas entre hombres y mujeres ya sea como herramientas educativas (Bonder, 2001), como estrategias de "empoderamiento" de grupos socialmente marginados (Bonder, 2006), como factor central para la planificación de programas destinados a la inclusión de sectores antes excluidos de los consumos tecnológicos (Bonder, 2008), como arena en la que se redefinirían constantemente lo público y lo privado como dimensiones que históricamente marcaron las relaciones de poder entre género (Causa, 2009), como medio para la movilización política feminista (Boix, 2002).

Otras autoras, atendiendo al impacto que las diferencias en la socialización de las mujeres podrían tener en su relación con bienes y conocimientos socialmente valorados, han señalado la tendencia a desalentar la relación delasmujeresconlastecnologías y con los campos de conocimiento tecnológico, especialmente en el ámbito educativo, tema que fue ampliamente investigado en la región latinoamericana (Natansohn, 2013; De Filippo, Sanz Casado y Gómez, 2009; Bonder, 2008).

En base a esta línea, surgieron estudios que problematizaron la presentación de la imagen de las mujeres en relación con las tecnologías, ya sea atendiendo a la manera en que esas imágenes tienden a naturalizar una relación de extrañeza y distancia con éstas, es el caso de la indagación respecto a las imágenes masculinas y femeninas masificadas por los videojuegos (Gros Salvat, 2002; Huertas Rojas, 2005), la manera en que se autorepresentan en foros virtuales las construcciones de etnia y género de las mujeres migrantes (da Silva, 2012; Huertas Bailén, 2012; Leung, 2007), la inclusión de mujeres en comunidades de programación y software libre (Pérez Bustos, Prieto y Franco Avellaneda, 2012) y la construcción de militancias feministas en espacios virtuales (Burkle y González, 2006).

La mayoría de los trabajos parten del reconocimiento de la situación de las mujeres como excluidas del ámbito de la tecnología y reclamando su inclusión, basándose en la asunción de la que la ausencia misma de las mujeres en el relato tecnológico es parte constitutiva de ésta como herramienta social y sistema de imaginarios asociados al prestigio, la razón y el poder.

Esta oclusión de la presencia de las mujeres en los análisis acerca de las relaciones sociales con la tecnología o las concepciones de la sociedad como 
tecnológica y la tecnología como social, constituye el planteo troncal de la propuesta del tecnofeminismo (Wajcman, 2006).

Desde una mirada que combina el feminismo cyborg y la teoría constructivista de la tecnología, dicha autora critica los enfoques dominantes en el campo científico de la investigación de las relaciones de tecnología y sociedad. La base de su crítica es su consideración del modo en que estas teorías han optado por interpretar la ausencia de mujeres o la ausencia de las marcas aparentes de presencia de las mujeres- en las áreas tecnológicas como argumento válido para afirmar que se trata de zonas libres de género, desgenerizadas, y por lo tanto ajenas a las relaciones de poder y de género que marcan todas las prácticas sociales. Siguiendo la misma línea de razonamiento, la presencia de cierto número de mujeres puede hacer que se interprete el campo de las tecnologías como igualitario, lo cual, al igual que en el caso anterior, deja fuera de discusión la política de género que es inherente a la existencia misma de la tecnología, según el pensamiento de Wajcman (Id., 2006).

A fin de integrar las relaciones de género en sus análisis de las dinámicas de innovación tecnológica, utiliza el concepto de relaciones sociales con las tecnologías en un sentido cercano al propuesto por Haraway. El mismo le permitió desplazar del centro de la discusión algunos determinismos que actuaban como limitantes de la mirada en esta área, por ejemplo las argumentaciones de larga data sobre si se puede o no considerar a las tecnologías como fuentes de cambios positivos o negativos por sí mismas, o si es necesario optar entre oponerse o apoyar los cambios tecnológicos.
Estas posiciones binarias suelen ser relativizadas y negadas en numerosas teorizaciones hegemónicas en torno a las tecnologías y la sociedad, pero son frecuentemente reconocibles en el centro mismo de sus argumentaciones en gran parte de los casos. En base a estas críticas la autora construye un enfoque autodenominado "tecnofeminista", en el cual las tecnologías son consideradas como parte del tejido social, una parte entre muchas otras, que en la época contemporánea ejercen un papel preponderante como pilares que aseguran la cohesión de las sociedades. De este modo, el cambio tecnológico y el cambio social ocurren de manera encadenada, en el plano material y en el significante. Las tecnologías digitales, en esta consideración, se definen como agentes contingentes y abiertos, que expresan las relaciones sociales en las que están integradas (Id., 2006).

De esta manera, el punto de partida del tecnofeminismo puede resumirse en la siguiente consideración: si la tecnología es parte integrante del entretejido social, dado que ella y la sociedad se producen mutuamente, es posible e indispensable traer a primer plano la manera en que las relaciones de poder generizadas interactúan con el diseño, la innovación y la valoración de las tecnologías, y la influencia diferencial del cambio tecnológico en agentes socializados de manera diferente de acuerdo a marcas y normativas de lo que se considera masculino o femenino.

Esta línea realiza valiosos aportes en el enfoque de estudios de género y tecnologías, al ubicar esa relación en el corazón de la reproducción y sostenimiento delos sistemastecnológico socialesactuales, visibilizar las relaciones inequitativas de los géneros con las tecnologías no como 
consecuencias sino como causas de la concentración de valor y prestigio que esa área detenta en los imaginarios actuales y proporcionar la base teórica para el análisis de la dimensión de las relaciones de género como inherentes al sistema tecno social. Del mismo modo, tiende un puente entre los estudios de género y los estudios de carácter socio técnico.

\section{CONCLUSIONES}

La sistematización crítica y analítica de algunos de los puntos de contacto interdisciplinar entre los campos de conocimiento de los Estudios de género y los Estudios sociales de la tecnología, nos permitió cumplir con dos objetivos principales de este artículo.

El primer de ellos fue la visibilización de la prolífica y variada producción teórica de diversas investigadoras y académicas inscriptas en el campo de los Estudios de género, quienes desarrollaron solventes dispositivos analíticos y metodológicos para llevar al terreno de los Estudios sociales de la tecnología, la reflexión acerca de los estatutos específicos del poder y de la diferencia característicos de los abordajes con perspectiva de género.

Mediante una exposición que abarcó el recorrido de investigaciones en tres ejes o concepciones teórico epistemológicas diferentes acerca de la tecnología, a saber, las perspectivas de las brechas, de las reformulaciones espacio-temporales, y los enfoques de la coproducción entre tecnología y sociedad, pudimos demostrar la presencia de producción académica con perspectiva de género en un arco amplio de las líneas de trabajo actualmente vigentes en los Estudios sociales de la tecnología.

Sostenemos que la sistematización de estas investigaciones, muchas veces invisibilizadas, y la puesta en diálogo de campos de conocimiento fácilmente percibidos como lejanos, resulta un aporte al avance de la inter, multi o transdisciplinariedad, entendida como imperativo en los contextos actuales producción de conocimiento académico.

\section{[VERONICA SOFIA FICOSECO]}

Doctora en Comunicación. Becaria post doctoral del Consejo Nacional de Investigaciones Científicas y Técnicas (CONICET -Argentina) y Universidad Nacional de Quilmes. Docente e investigadora en la Universidad Nacional de la Patagonia Austral. 


\section{REFERÊNCIAS BIBLIOGRÁFICAS}

AMORÓS, Celia. Mujeres e imaginarios de la globalización. Reflexiones para una agenda teórica global del feminismo. Rosario: Homo Sapiens Ediciones, 2008.

BONDER, Gloria. Las nuevas tecnologías de información y las mujeres: reflexiones necesarias. In: REUNION DE EXPERTOS SOBRE GLOBALIZACION, CAMBIO TECNOLOGICO Y GENERO. Sao Paulo: 2001.

Género en acción en la sociedad del conocimiento: oportunidades para la innovación. SEMINARIO INTERNACIONAL LA SOCIEDAD DE LA INFORMACION EN LA COOPERACION AL DESARROLLO. España: Fundación Tres Culturas del Mediterráneo, 2006.

Juventud, género y TIC: imaginarios en la construcción de la sociedad de la información en América Latina. In: ARBOR. Ciencia, pensamiento y cultura. Madrid: 2008.

BOIX, Monserrat. Comunicación, Feminismo y Nuevas Tecnologías. En: Aportes Andinos No.4. Ecuador: Universidad Andina Simón Bolívar, 2002.

BURKLE, Eva y GONZALEZ, Martha "Propuesta metodológica para el análisis deplataformas en Internet: El caso de las redes de mujeres". En: Revista Razón y Palabra. México: ITESM, No. 49, año 2006.

CASTAÑO, Cecilia: La segunda brecha digital. Cátedra. Madrid: Cátedra, 2008.

CASTAÑO, Celia, MARTIN, Juan y Vázquez, Susana. La brecha digital de género: acceso, uso y habilidades. In: CASTAÑO, Cecilia: La segunda brecha digital. Madrid: Cátedra, 2008 .

CAUSA, Adriana. Genero, pobreza y Tecnologías. Travesías complejas de las mujeres ante la apropiación de las TICs. In: Margen revista de Trabajo Social. Buenos Aires: No. $54,2009$.

DE ABREU, Carla Luzia. Apuntes sobre las identidades de géneros y de sexualidades nonormativas en las redes sociales virtuales. In: CONGRESO INTERNACIONAL LAS POLITICAS DE EQUIDAD DE GENERO EN PROSPECTIVA. Nuevos escenarios, actores y articulaciones. Buenos Aires: FLACSO, 2010.

DE FILIPPO, Daniela, SANZ CASADO, Elías y GÓMEZ, Isabel. Movilidad científica y género. Estudio del profesorado de una universidad española. In: Revista Mexicana de Sociología México: Universidad Nacional Autónoma de México-Instituto de Investigaciones Sociales, 71, número 2, 2009.

GIACOSA, María Ester, SOSA, Carolina. Y ZALDÚA, Analía. Las NTICs y su impacto en 
la educación a distancia y la comunicación. In: XIII JORNADAS DE INVESTIGADORES EN COMUNICACIÓN. San Luis: 2009.

GROS SALVAT, Begoña. La dimensión socioeducativa de los videojuegos. En: Revista electrónica de Tecnología Educativa. Barcelona: Departamento de Teoría e Historia de la Educación, Universitat de Barcelona, No. 12, 2001.

HARAWAY, Donna. Ciencia, cyborgs y mujeres. La reinvención de la naturaleza. Madrir: Cátedra, 1995.

Las promesas de los monstruos. Una política regeneradora para otros inapropiados/bles. En: Revista Política y Sociedad. Madrid: No. 30, 1999.

HUERTAS ROJAS, Fernando. La violencia virtual. Una experiencia de los jóvenes en lasvideosalas. In: Estudios Sociales Revista de investigación científica. México: Volumen 13, 2005.

LAGO MARTINEZ, Silvia. Las políticas gubernamentales para la sociedad de la información en Argentina. In: FINQUELIEVICH, Silvia (coord.). E-política y e-gobierno en América Latina. Buenos Aires: 2005, http://www.links.org.ar/infoteca/E-Gobiernoy-E-Politica-en-LATAM.pdf.

LEUNG, Linda. Etnicidad Virtual. Raza, resistencia y world wide web. Barcelona: Gedisa, 2007.

NATANSOHN, Graciela. Internet en código femenino. Teorías y prácticas. Buenos Aires: La Crujía, 2013. 151 p.

PEREZ BUSTOS, Tania; PRIETO, Fabián y FRANCO AVELLANEDA, Manuel. Una lectura de las tecnologías desde los estudios feministas. El caso OLPC y Sugar Labs en Colombia. In: Revista Nómadas. Colombia: Universidad Central de Colombia, No. 36, 2012.

PLANT, Sadie. Ceros + unos. Mujeres digitales + la nueva tecnocultura. España: Editorial Destino, 1998.

SAINZ, Milagrosy GONZALEZ, Ana. La segunda brecha digital:educación einvestigación”. In: CASTAÑO, Cecilia: La segunda brecha digital. Madrid: Cátedra. 2008.

SALLÉ, María Ángeles. Las políticas de la sociedad de la información en igualdad y para la igualdad". In: CASTAÑO, Cecilia: La segunda brecha digital. Madrid: Cátedra, 2008.

STONE, Allucquere Rossane. Will the Real Body Please Stand Up? Boundary Stories About Virtual Cultures. In: Cybersexualities. Escocia: Edinburgh University Press, 1999.

VAZQUEZ, Susana y AÑINO, Sara. La diversidad de las mujeres ante Internet. In: CASTAÑO, Cecilia. La segunda brecha digital. Madrid: Cátedra, 2008.

WAJCMAN, Judy. El tecno feminismo. Madrid: Cátedra, 2006. 